\title{
Disposição cinética do atenolol em pacientes coronarianos submetidos a revascularização do miocárdio
}

\author{
Fátima da Silva Leite ${ }^{1}$, Andreia Zago Chignalia', Maria José Carvalho Carmona ${ }^{2}$, \\ José Otávio Costa Auler Junior², Silvia Regina Cavani Jorge Santos ${ }^{1, *}$
}

${ }^{1}$ Faculdade de Ciências Farmacêuticas da Universidade de São Paulo, ${ }^{2}$ Instituto do Coração, HCFMUSP

*Correspondence:

S. R. C. J. Santos

Laboratório de Farmacologia e

Terapêutica

Departamento de Farmácia

Faculdade de Ciências Farmacêuticas

Universidade de São Paulo

Av. Prof Lineu Prestes, 580

05508-000 - São Paulo, SP - Brasil

E-mail: pharther@usp.br
A isquemia miocárdica é um importante fator de risco para a mortalidade e eventos cardiovasculares no perioperatório de cirurgias cardíacas e não-cardíacas, sendo que a administração profilática de $\beta$-bloqueadores nesse periodo, reduz estes riscos. Sabe-se que alterações fisiológicas ocorridas durante a cirurgia de revascularização do miocárdio (RM) com circulação extracorpórea (CEC) podem afetar as concentrações plasmáticas e a cinética de muitos fármacos. Neste estudo, investigou-se a farmacocinética do atenolol em pacientes com angina instável e sem prejuízo renal, submetidos à revascularização com CEC e em terapia crônica com atenolol peroral. O estudo farmacocinético exigiu coleta de amostras sangüineas seriadas após as doses prée pós-operatória. Comparado ao pré-operatório, registrou-se redução não significativa no volume de distribuição e na depuração plasmática após a cirurgia, permanecendo inalterada a meia-vida biológica $(p>0,05)$. Uma correlação linear negativa entre meiavida e depuração pode ser estabelecida nos dois períodos do estudo ( $r:-0,77, p=0,06$ no pré-operatório e $r:-0,89, p=0,06$ no pósoperatório), enquanto que se estimou correlação linear direta entre volume de distribuição e meia-vida biológica apenas no précirúrgico ( $r: 0,54, p=0,03$ no pré-operatório e $r: 0,09, p=0,03$ no pós-operatório). Conclui-se que a cirurgia de revascularização auxilia no restabelecimento da extensão da distribuição do atenolol.
Uniterms

- Atenolol

- Farmacocinética

- Revascularização do miocárdio (RM)

- Circulação extracorpórea (CEC)

\section{INTRODUÇÃO}

O procedimento cirúrgico desencadeia intensa estimulação adrenérgica, aumentando o risco de isquemia miocárdica perioperatória, responsável pelo aumento da mortalidade e de eventos cardiovasculares no pós-opera- tório. Em contrapartida, a administração profilática dos $\beta$ bloqueadores, nos períodos pré- e pós-operatório, reduz a morbidade e a mortalidade de pacientes com insuficiência coronariana submetidos a cirurgias não-cardíacas (Mangano et al., 1996; Wallace et al., 1998) e cardíacas (Ferguson et al., 2002). 
O propranolol é um $\beta$-bloqueador lipofílico não-seletivo largamente utilizado devido a seu baixo custo. Entretanto, estudos relatados na literatura sugerem que a dose de propranolol administrada no período pós-operatório de cirurgia cardíaca deva ser reduzida quando comparada com a dose utilizada no pré-operatório, devido ao risco de depressão do miocárdio, causada pelo procedimento cirúrgico com circulação extracorpórea e hipotermia, sobre as concentrações plasmáticas e a farmacocinética do propranolol (Mets, 2000; Carmona et al., 2005).

$\mathrm{O}$ atenolol é empregado como fármaco de primeira escolha no tratamento de doenças cardiovasculares, por ser um fármaco cardiosseletivo e de longa duração de ação. Administrado oralmente, o atenolol mostra-se efetivo no tratamento de angina pectoris e outras doenças coronarianas isquêmicas, reduzindo a gravidade e a freqüência de ataques isquêmicos, o consumo de nitroglicerina e melhorando a função cardiovascular durante o exercício. Além disso, este agente $\beta$-bloqueador pode ser administrado com segurança, devido ao alto índice terapêutico (Wadworth et al., 1991).

Em relação à farmacocinética do atenolol, a literatura relata que para indivíduos saudáveis apenas $50 \%$ da dose são absorvidos pelo trato gastrintestinal após a administração peroral. Uma vez atingida a corrente circulatória, o atenolol é rapidamente distribuído para os tecidos devido à sua baixa ligação às proteínas plasmáticas (ao redor de $5 \%$ ), embora o mesmo apresente uma extensão de distribuição relativamente baixa $(0,95 \mathrm{~L} / \mathrm{kg})$. Devido a sua característica hidrofílica, a eliminação do atenolol é predominantemente renal, sendo que a fração da dose absorvida é recuperada na urina dentro de 48 horas, apresentando meia-vida biológica de 4 a 8 horas e depuração plasmática de 1,8 a 2,2 mL/min.kg. (Wadworth et al., 1991; Mason et al., 1979). Em virtude de sua excreção função renal dependente, verifica-se que o atenolol tende a se acumular em pacientes com insuficiência renal e a dose deve ser ajustada de acordo com grau de disfunção renal. (Hoffman, 2001).

Entretanto, relata-se que a disposição cinética do atenolol mostra-se alterada em pacientes com angina estável induzida pelo exercício, tratados cronicamente com atenolol $\mathrm{PO}$, visto que estes pacientes apresentam um prolongamento da meia-vida biológica ao redor de 2 vezes (11 horas), decorrente do aumento do volume aparente de distribuição $(2,02 \mathrm{~L} / \mathrm{kg}$ e 2,12 L/kg, respectivamente, para as doses de 100 e $200 \mathrm{mg} /$ dia). A depuração plasmática do atenolol neste grupo de paciente é de cerca de 2,2 mL/min.kg. (Majid et al., 1979).

Desta forma, o objetivo deste estudo é o de comparar a farmacocinética do atenolol nos períodos pré- e pós-operatório de cirurgia cardíaca, em pacientes coronarianos porta- dores de angina instável, submetidos à revascularização do miocárdio com circulação extracorpórea.

\section{MATERIAL E MÉTODO}

\section{Protocolo clínico}

Este protocolo foi previamente aprovado pelos Comitês de Ética em Pesquisa das instituições envolvidas (Faculdade de Ciências Farmacêuticas /USP e Instituto do Coração/HC FMUSP). Além disso, todos os pacientes participantes do protocolo de pesquisa forneceram o Termo de Consentimento Livre e Esclarecido após serem informados em detalhes sobre os procedimentos a serem realizados durante o estudo.

Este estudo aberto, cruzado e rigorosamente controlado foi realizado em duas fases. Avaliaram-se seis pacientes coronarianos do sexo masculino portadores de insuficiência coronariana grave, submetidos à cirurgia eletiva de revascularização do miocárdio com circulação extracorpórea e hipotermia e em terapia crônica com atenolol PO a pelo menos uma semana. Os pacientes estavam sendo tratados antes da cirurgia com doses de atenolol variando entre 25 e $100 \mathrm{mg}$ ao dia. O esquema posológico do atenolol para os pacientes incluídos neste estudo encontra-se descrito a seguir: $25 \mathrm{mg}$, uma vez ao dia (paciente AFO); $50 \mathrm{mg}$, uma vez ao dia (pacientes S-T e FLA); $50 \mathrm{mg}$, duas vezes ao dia (pacientes AACF; N-M e JOGR).

Todos os pacientes incluídos no estudo apresentavam funções renal, hepática e endócrina dentro dos limites da normalidade. Além disso, foram excluídos pacientes com idade superior a 65 anos, obesidade mórbida, indivíduos com fração de ejeção do ventrículo esquerdo inferior a $35 \%$, com sorologia positiva para hepatite, pacientes nefrectomizados ou com creatinina sérica maior que $1,4 \mathrm{mg} / \mathrm{dL}$, atividade de protrombina inferior a $80 \%$ e uso de anticoagulantes orais. Os dados antropométricos dos pacientes avaliados neste estudo estão resumidos na Tabela I. No dia anterior à cirurgia, os pacientes incluídos no estudo foram avaliados em relação aos exames laboratoriais da rotina pré-operatória e ao risco cirúrgico segundo o critério proposto por Higgins (Higgins et al., 1992).

Posteriormente à avaliação inicial, a última dose da medicação em estudo foi administrada no dia que precedeu o procedimento cirúrgico e procedeu-se à coleta de seis amostras sanguíneas seriadas, de $3 \mathrm{~mL}$ cada, através de punção venosa, em tubos contendo anticoagulante EDTA, nos seguintes períodos: zero (imediatamente antes a administração do $\beta$-bloqueador) e 2, 4, 6, 8 e 12 horas após a ingestão da dose peroral pré-operatória de atenolol. Na manhã do dia seguinte, os pacientes foram submetidos 
TABELA I - Caracterização dos pacientes avaliados no estudo

\begin{tabular}{lcccccc}
\hline Iniciais & Idade (anos) & Peso $(\mathrm{kg})$ & Altura $(\mathrm{m})$ & $\mathrm{IMC}\left(\mathrm{kg} / \mathrm{m}^{2}\right)$ & $\begin{array}{c}\text { Superfície } \\
\text { Corporal }\left(\mathrm{m}^{2}\right)\end{array}$ & $\begin{array}{c}\text { Risco } \\
\text { cirúrgico }\end{array}$ \\
\hline AFO & 52 & 72 & 1,72 & 24,34 & 1,90 & 3 \\
S-T & 59 & 82 & 1,70 & 28,37 & 1,95 & 0 \\
AACF & 64 & 70 & 1,70 & 24,22 & 1,80 & 1 \\
FLA & 62 & 69 & 1,66 & 25,15 & 1,80 & 0 \\
N-M & 64 & 96 & 1,67 & 34,42 & 2,20 & 2 \\
JOGR & 61 & 92 & 1,72 & 31,10 & 2,00 & 3 \\
Média & 60,33 & 80,17 & 1,70 & 27,93 & 1,94 & 1,5 \\
DP & 4,11 & 10,71 & 0,02 & 3,80 & 0,14 & 1,26 \\
\hline
\end{tabular}

Dados expressos em médias e desvio padrão (DP).

à cirurgia de revascularização do miocárdio com circulação extracorpórea.

Ao final do período pós-operatório imediato, após estabilização hemodinâmica e extubação do paciente, o $\beta$-bloqueador foi re-introduzido e os indivíduos avaliados receberam uma dose peroral de atenolol de $25 \mathrm{mg}$ (pacientes AFO; S-T e FLA) ou 12,5 mg (pacientes AACF; N-M e JOCR). Posteriormente, foram coletadas sete amostras sangüíneas seriadas de $3 \mathrm{~mL}$ cada, nos seguintes tempos: zero (imediatamente antes a administração do fármaco) e 2, 4, 6, 8, 12 e 24 horas após a administração da dose.

Ressalta-se, ainda, que um volume total inferior a $40 \mathrm{~mL}$ foi coletado de cada paciente avaliado, em ambos períodos do estudo.

\section{Metodologia Analítica}

Após a coleta, as amostras sanguíneas foram centrifugadas e o plasma foi congelado a $-20{ }^{\circ} \mathrm{C}$ até o momento da análise. Utilizou-se o método relatado anteriormente, empregando a cromatografia líquida de alta eficiência com detecção por fluorescência (CLAE-F), para a quantificação do atenolol nas amostras de plasma (Leite et al., 2006).

\section{Análise Farmacocinética}

A disposição cinética do atenolol foi avaliada nos períodos pré e pós-operatório através da curva de decaimento ( $\mathrm{C}$ versus $\mathrm{t}$ ), em que a transformação logarítmica das concentrações plasmáticas foram plotadas em função dos tempos de coleta. De acordo com o protocolo de coletas, optou-se pela utilização do modelo de um compartimento aberto para fármacos com administração peroral e em regime de dose múltipla. A modelagem farmacocinética foi realizada através da utilização do software PK Solutions 2.0 Noncompartmental
Pharmacokinetics Data Analysis (Ashland, OH, USA.), para a estimativa dos parâmetros farmacocinéticos modelo-dependentes (constante de velocidade de eliminação e meia-vida biológica) e modelo-independentes (depuração plasmática e volume aparente de distribuição).

\section{Análise Estatística}

Para comparação dos parâmetros farmacocinéticos obtidos nos períodos pré e pós-operatório, aplicou-se estatística não-paramétrica (teste de Wilcoxon), através da utilização do software GraphPad 2.01, GraphPad Software Incorporated (San Disgo, USA). Adicionalmente, para a obtenção das correlações entre parâmetros farmacocinéticos estimados, aplicou-se o teste de Spearman utilizando o software GraphPad Instant 2.01. Os resultados da farmacocinética foram expressos através da média, mediana e limites superior e inferior da faixa de variação IC 95\%. Os demais resultados obtidos foram expressos através da média e desvio padrão.

\section{RESULTADOS}

O período pré-operatório foi definido no protocolo de estudo como o dia que precedeu a cirurgia cardíaca, sendo que o intervalo entre a última dose do $\beta$-bloqueador e o início da cirurgia foi de 1025,00 $\pm 367,26$ horas (média e DP). Subseqüentemente, o pós-operatório geralmente ocorreu a partir da $18^{a}$ hora do término da cirurgia, iniciando-se com a extubação do paciente, quando o mesmo apresenta-se estável hemodinamicamente para a re-introdução de toda a medicação peroral, incluindo o atenolol. Destaca-se, ainda, que procedimento cirúrgico foi realizado num tempo médio de $357,50 \pm 50,57$ minutos, enquanto que o tempo de circulação extracóporea foi, em média de $76,67 \pm 23,17$ minutos. 
A Figura 1 apresenta as transformações logarítmicas das concentrações plasmáticas do atenolol, obtidas em função dos tempos de coletas, nos períodos pré- e pós-operatório da cirurgia cardíaca com circulação extracorpórea, representadas através das medianas. Com base nestes dados, é possível observar que as curvas de decaimento são paralelas, indicando que a velocidade de eliminação plasmática do atenolol é semelhante nos dois períodos avaliados nos respectivos intervalos de dose.

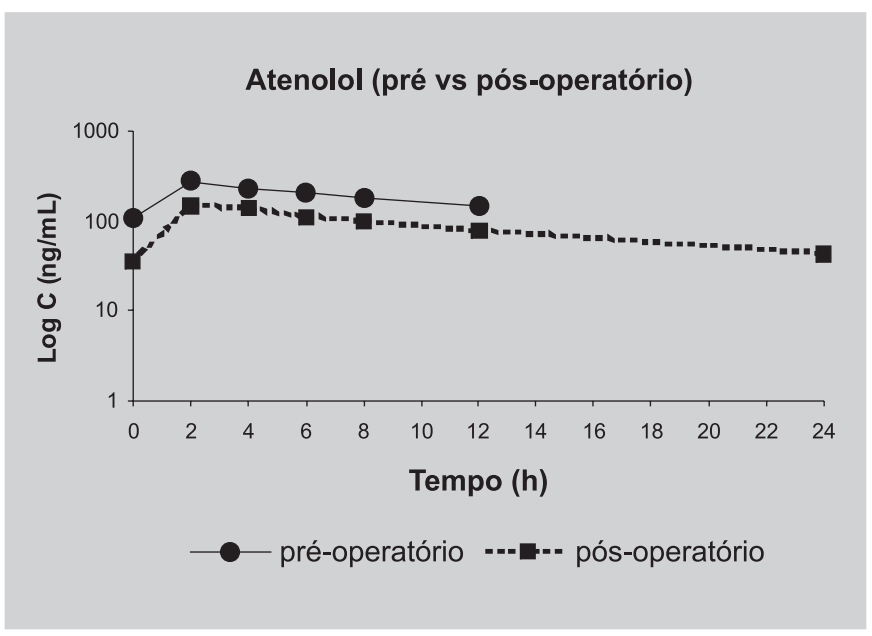

FIGURA 1 - Curvas de decaimento plasmático do atenolol no intervalo de tempo nos períodos pré $(0-12 \mathrm{~h})$ e pósoperatório (0-24 h) de cirurgia cardíaca. Valores expressos em medianas.

Os parâmetros farmacocinéticos do atenolol (meiavida biológica, constante de eliminação, depuração plasmática e volume aparente de distribuição), obtidos nos períodos pré-operatório e após a revascularização do miocárdio com circulação extracorpórea estão resumidos na Figura 2.

Apesar da redução em cerca de duas vezes no volume aparente de distribuição do atenolol no período pósoperatório, registrou-se variação não significativa, em função da maior variabilidade encontrada no pós-operatório, relativamente ao dado estimado previamente à cirurgia de revascularização, conforme segue: $1,02(0,35-$ $1,93) \mathrm{L} / \mathrm{kg}$ versus $1,92(0,99-2,39) \mathrm{L} / \mathrm{kg}, \mathrm{p}=0,22(\mathrm{NS})$, (medianas e limites inferior e superior IC95\%). Os resultados obtidos para a depuração plasmática, expressos através das medianas e IC95\%, registram, ainda, ligeira redução $(p=0,31, N S)$ no parâmetro farmacocinético após a cirurgia cardíaca $(1,32 ; 0,34-2,34 \mathrm{~mL} / \mathrm{min} . \mathrm{kg})$, quando comparado ao período pré-cirúrgico $(1,76 ; 0,84$ $3,09 \mathrm{~mL} / \mathrm{min} . \mathrm{kg}$ ).

Por outro lado, os parâmetros farmacocinéticos modelo-dependentes permaneceram praticamente inalterados no pós-operatório relativamente ao pré-operatório, conforme segue: (meia-vida biológica: 13,54 vs 11,66 h; constante de eliminação: 0,052 vs $0,059 \mathrm{~h}^{-1} \mathrm{p}=0,56$, medianas). Tal resultado pode ser justificado pelo fato da depuração plasmática e do volume aparente de distribuição apresentarem a mesma tendência no pós-operatório, quando comparado aos valores obtidos antes da cirurgia cardíaca.

A Figura 3 ilustra a correlação linear entre os parâmetros farmacocinéticos obtidos nos períodos pré- e pósoperatório de cirurgia de revascularização do miocárdio.

Registrou-se boa correlação linear inversa entre a

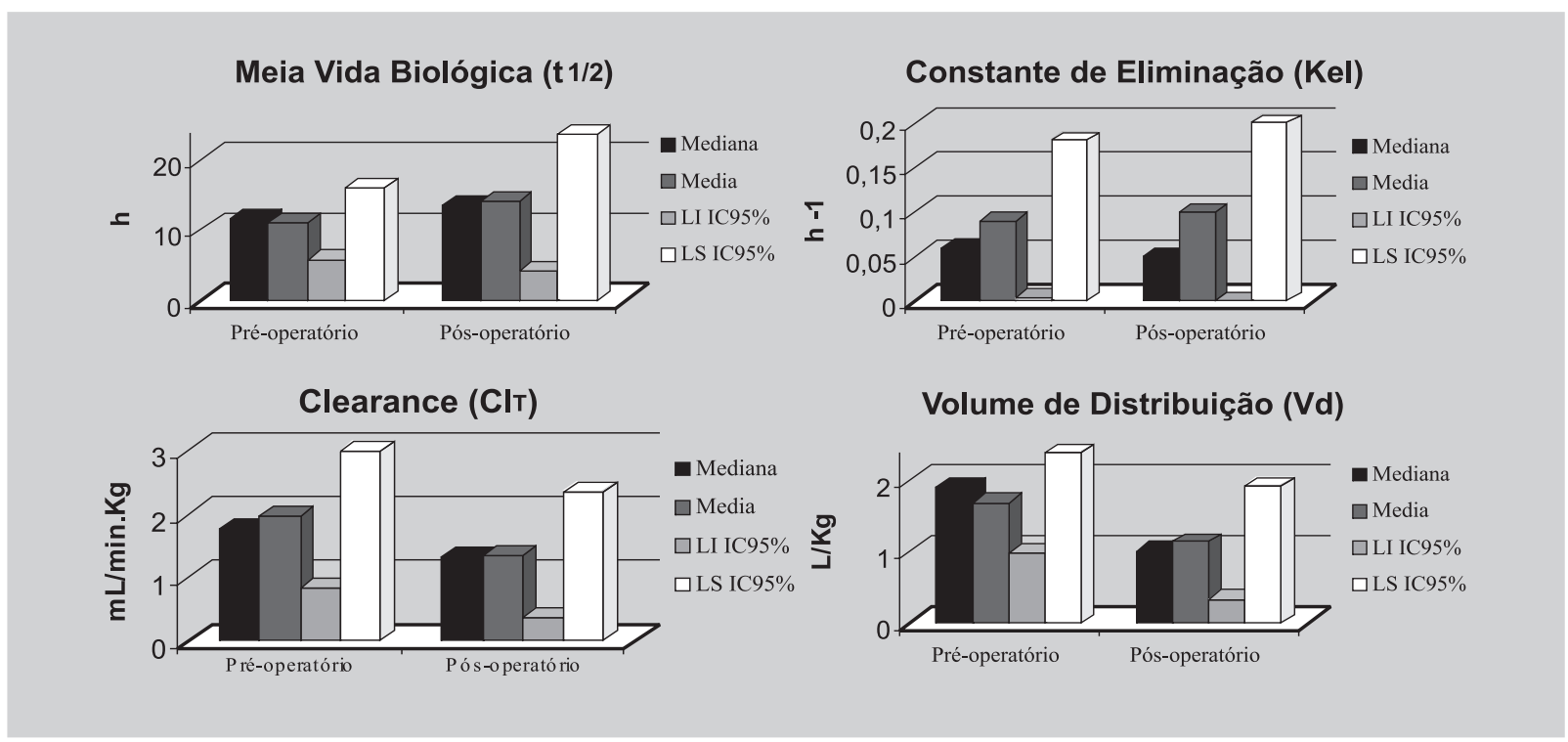

FIGURA 2 - Parâmetros farmacocinéticos do atenolol em pacientes avaliados nos períodos pré- e pós-operatório de cirurgia cardíaca. Valores expressos em médias, medianas, limites inferior e superior da faixa de variação IC $95 \%$. 


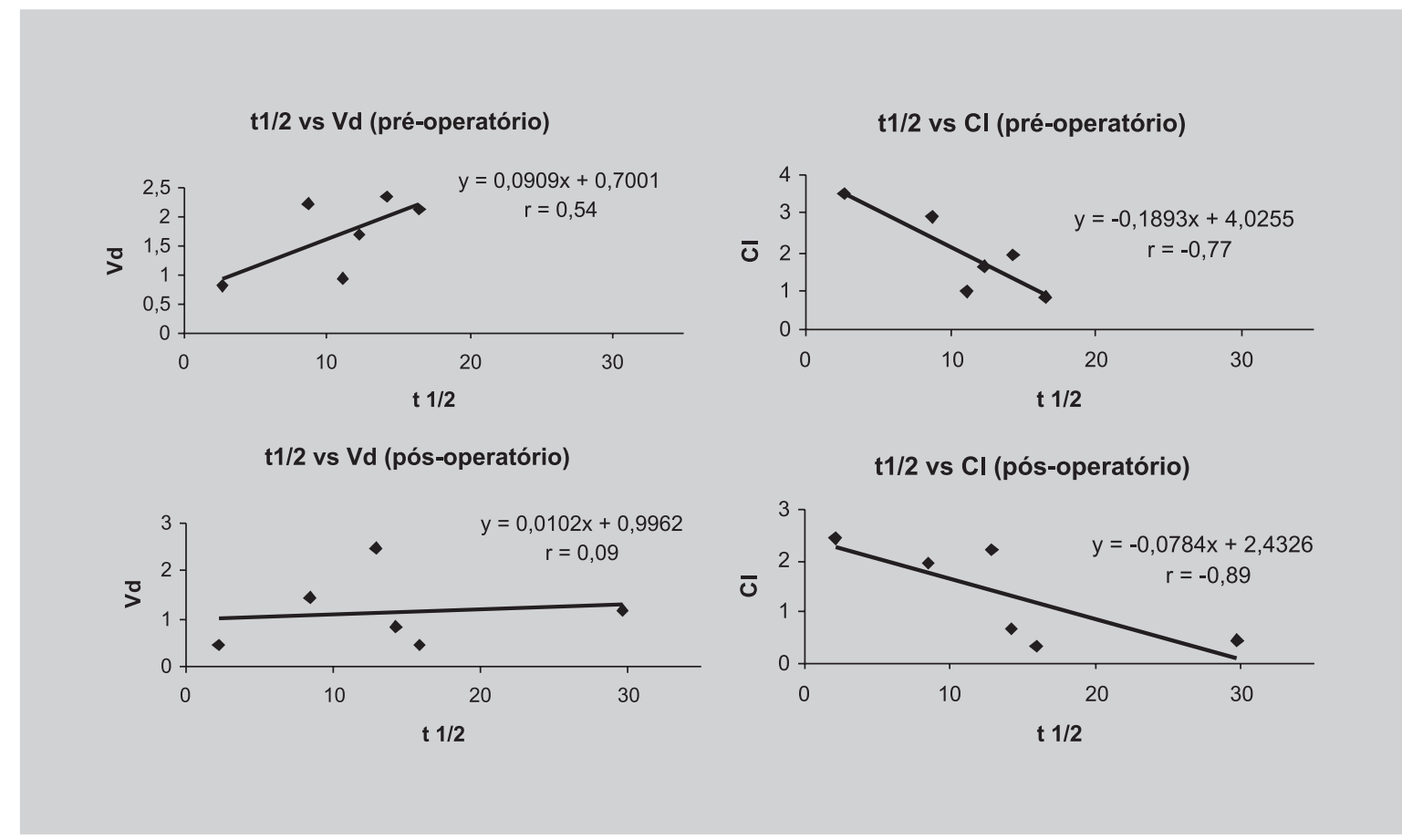

FIGURA 3 - Correlação linear (teste de Spearman) entre os parâmetros farmacocinéticos obtidos nos períodos pré- e pós-operatório de revascularização do miocárdio.

depuração plasmática e a meia-vida biológica do atenolol no pré-operatório (r: - 0,77 ), indicando que o prolongamento da meia-vida varia na proporção inversa da depuração plasmática. Esta correlação aumenta após a revascularização do miocárdio com circulação extracorpórea (r: - 0,88) em virtude da melhora da função cardíaca do paciente após a cirurgia.

Por outro lado, foi possível estabelecer apenas no pré-operatório uma correlação razoável $(\mathrm{r}: 0,54)$ entre a meia-vida biológica e o volume aparente de distribuição. A falta de correlação entre esses parâmetros no período pós-operatório pode ser justificada pelas alterações que ocorrem com melhora hemodinâmica após a revascularização do miocárdio (r: 0,09).

\section{DISCUSSÃO}

O procedimento de circulação extracorpórea pode causar alterações importantes nos níveis plasmáticos e na disposição cinética de muitos fármacos, em virtude de alterações hemodinâmicas decorrentes da hemodiluição, hipotermia, fluxo sanguíneo não-pulsátil, além da anticoagulação, isolação dos pulmões da circulação e pelo seqüestro de alguns fármacos pelo equipamento de CEC (Mets, 2000; Holley et al., 1982). Relata-se prolongamento de 2,5 vezes na meia-vida biológica e aumento de duas vezes no volume de distribuição para o $\beta$-bloqueador lipofílico propranolol, além da manutenção de altas concentrações plasmáticas logo após a cirurgia de revascularização do miocárdio com CEC e hipotermia (Carmona et al., 2005).

Entretanto, para alguns fármacos hidrofílicos de excreção renal, tais como alguns antimicrobianos de administração profilática como, por exemplo, a cefuroxima, estas alterações muitas vezes mostram-se irrelevantes para pacientes com a função renal dentro da normalidade (dados não publicados). Desta forma, seria de se esperar que as alterações nos parâmetros farmacocinéticos do atenolol decorrentes do procedimento cirúrgico com CEC não sejam tão significativas, quando comparadas às modificações reportadas para fármacos lipofílicos.

No presente estudo aberto, cruzado e rigorosamente controlado, investigaram-se seis pacientes portadores de angina instável grave e indicação cirúrgica de revascularização do miocárdio com circulação extracorpórea. Em todos os pacientes investigou-se a farmacocinética do atenolol nos períodos pré- e pós-operatório. Os dados obtidos neste estudo evidenciam redução do volume aparente de distribuição da ordem de duas vezes nos pacientes pós-cirúrgicos, quando comparado com os valores estimados antes da cirurgia de revascularização do miocárdio. Entretanto, verifica-se que esta redução no parâmetro farmacocinético não foi estatis- 
ticamente significativa, devido à maior variabilidade do parâmetro registrada após a cirurgia cardíaca.

Apesar da ausência de significância estatística, os dados obtidos neste estudo sugerem que a cirurgia de revascularização auxilia no restabelecimento da função cardíaca do paciente, com conseqüente melhora hemodinâmica, responsável pela normalização da extensão de distribuição do atenolol, visto que os valores estimados para o volume aparente de distribuição antes do procedimento cirúrgico são concordantes com os valores relatados anteriormente para pacientes com angina estável induzida pelo exercício (Majid et al., 1979) e que os resultados obtidos após a intervenção cirúrgica são semelhantes aos valores relatados na literatura para indivíduos saudáveis (Wadworth et al., 1991).

Em relação à depuração plasmática, verifica-se que os resultados obtidos para este parâmetro farmacocinético antes da cirurgia de revascularização são comparáveis aos valores relatados na literatura para indivíduos saudáveis; porém, como já era esperado, a depuração plasmática do atenolol encontra-se ligeiramente reduzida $(34 \%$, NS) no período pós-operatório dos pacientes investigados nesse estudo, em virtude dos efeitos causados pelo procedimento cirúrgico com CEC (Mets, 2000; Holley et al., 1982), visto que durante o procedimento de circulação extracorpórea, há a redução da perfusão periférica, com conseqüente redução da perfusão renal e diminuição da depuração plasmática do fármaco.

Finalmente, constatou-se, ainda, que em relação aos voluntários sadios, a meia-vida biológica mostra-se prolongada em cerca de duas vezes, em ambos os períodos do estudo, em decorrência do aumento registrado no volume de distribuição antes da cirurgia cardíaca de revascularização e ligeira redução da depuração plasmática do atenolol no período pós-cirúrgico.

Além disso, o estudo de correlação entre os parâmetros farmacocinéticos investigados neste estudo demonstra a existência de correlação linear entre volume aparente de distribuição e a meia-vida biológica do atenolol apenas no período pré-cirúrgico, sendo que após a revascularização do miocárdio, não é possível estabelecer correlação entre estes dois parâmetros. Em relação à depuração plasmática, observa-se que a meiavida biológica apresenta boa correlação com o clearance plasmático do fármaco nos dois períodos de estudo, sendo que esta correlação melhora no período pós-operatório (r: -0,77 para r: -0,88); sugerindo que no período que antecede a cirurgia cardíaca, a meia-vida biológica do fármaco depende da extensão de distribuição e da depuração plasmática, enquanto que após a revascularização, a eliminação do atenolol é função apenas da depuração plasmática, uma vez que a cirurgia de revascularização é responsável pelo restabelecimento da função cardíaca do paciente portador de insuficiência coronariana e que após a correção cirúrgica, há a reconstituição dos compartimentos fluidos e da função hemodinâmica do paciente, com conseqüente normalização da extensão da distribuição do atenolol.

\section{CONCLUSÃO}

Com base nos resultados obtidos, observa-se que apesar da alta variabilidade nos parâmetros farmacocinéticos estimados, observa-se tendência de normalização do volume aparente de distribuição do atenolol após a cirurgia cardíaca de revascularização do miocárdio. Além disso, o estudo de correlação entre os parâmetros farmacocinéticos investigados nos permite sugerir que antes da intervenção cirúrgica, as concentrações plasmáticas do atenolol no paciente coronariano dependem da depuração plasmática e da extensão da distribuição do fármaco, enquanto as concentrações plasmáticas após o procedimento de revascularização do miocárdio é função apenas da sua depuração plasmática, visto que a função hemodinâmica do paciente revascularizado é restabelecida após a correção cirúrgica.

\section{ABSTRACT}

\section{Kinetic disposition of atenolol in coronary patients submitted to the CABG surgery}

Myocardium ischemia is an important factor of risk for mortality and cardiovascular events in the perioperative period of cardiac and non cardiac surgeries. However, the prophylactic administration of $\beta$-blocker agents could reduce these risks. Physiologic changes, occurred during the coronary artery bypass graft (CABG) surgery with cardiopulmonary bypass (CPB), could alter plasma concentration and pharmacokinetics of many drugs. This study investigated the pharmacokinetics of atenolol in patients with unstable angina and without renal dysfunction, submitted to CABG surgery and treated chronically with atenolol PO. For pharmacokinetic analysis, 13 blood samples were collected after doses administrated pre-and post-operatively. Compared to the pre-operative period, it was verified a nonsignificant reduction in the apparent volume of distribution and plasma clearance after the surgery, remaining unchanged the biological half-life, $p>0.05$ (NS). A negative linear correlation between plasma 
clearance and elimination half-life was demonstrated in both periods of the study ( $r:-0.77 p=0.06$, pre-surgery and $r:-0.89, p=0.06$, post-surgery), while a correlation between volume of distribution and biological half-life was established only before revascularization ( $r: 0,54$ $p=0,03$, pre-surgery and $r: 0,09, p=0,03$, post-surgery). We suggest that the CABG surgery leads to the normalization of the extension of distribution of atenolol.

UNITERMS: Atenolol. Pharmacokinetics. Coronary artery bypass graft (CABG). Cardiopulmonary bypass (CPB).

\section{AGRADECIMENTOS}

Fundação de Amparo à Pesquisa do Estado de São Paulo (FAPESP) e Conselho Nacional de Desenvolvimento Científico e Tecnológico (CNPq), pelo suporte financeiro.

\section{REFERÊNCIAS BIBLIOGRÁFICAS}

CARMONA, M.J.C.; MALBOUISSON, L.M.S.; PEREIRA V.A.; BERTOLINE, M.A.; OMOSAKO C.E.K.; LE BIHAN, K.B.; AULER JR, J.O.C.; SANTOS, S.R.C.J. Cardiopulmonary bypass alters the pharmacokinetics of propranolol in patients undergoing cardiac surgery. Braz. J. Med. Biol. Res., v. 38, n. 5, p. 713-721, 2005.

FERGUSON, T.B.; COOMBS, L.P.; PETERSON, E.D. Preoperative b-blocker use and mortality and morbidity following CABG surgery in North America. Jama, v. 287, n. 17, p. 2221-2227, 2002.

HIGGINS, T.L.; ESTAFANOUS, F.G.; LOOP, F.D.; BECK, G.J.; BLUM, J.M.; PARANANDI, L. Stratification of morbidity and mortality outcome by preoperative risk factors in coronary artery bypass patients. A clinical severity score. J. Am. Med. Assoc., v. 267, p. 2344-2348, 1992.

HOFFMAN B.B. Catecholamines, sympathomimetic drugs, and adrenergic receptor antagonists. In: HARDMAN J.G.; LIMBIRD L.E.; GILMAN A.G., eds, Goodman \& Gilman's The Pharmacological Basis of Therapeutics. 10 ed. New York: McGraw-Hill, cap.10, p. 215-268, 2001.
HOLLEY, F.O.; PONGANIS, K.V.; STANSKI, D.R. Effect of cardiopulmonary bypass on the pharmacokinetics of drugs. Clin. Pharmacokinet., v. 7, p. 234-251, 1982.

LEITE, F.S.; PEREIRA, V.A.; OMOSAKO, C.E.; CARMONA, M.J.C.; AULER JR, J.O.C.; SANTOS, S.R.C.J.A micromethod for the quantification of atenolol in plasma using high-performance liquid chromatography with fluorescence detection. Therapeutic drug monitoring of two patients with severe coronary insufficiency before cardiac surgery. Ther. Drug Monit., v. 28, n. 2, p. 237-244, 2006.

MAJID, P.A.; WAN DER VIJGH, W.J.; DE FEIJTER, P.J.; WARDEH, R.; WAN DER WALL, E.E.; ROOS, J.P. Once daily atenolol (Tenormine) in the treatment of angina pectoris. Observations on clinical efficacy, pharmacokinetics and pharmacodynamics. Eur. J. Cardiol., v. 9, n. 6, p. 419-435, 1979.

MANGANO, D.T.; LAYUG, E.L.; WALLACE, A.; TATEO, I. Effect of atenolol on mortality and cardiovascular morbidity after noncardiac surgery. N. Engl. J. Med., v. 335, n. 23, p. 1713-1720, 1996.

MASON, W.D.; WINER, N.; KOCHAK, G.; COHEN, I.; BELL, R. Kinetics and absolute bioavailability of atenolol. Clin. Pharmacol. Ther., v. 25, n. 4, p. 408-415, 1979.

METS, B. The pharmacokinetics of anesthetic drugs and adjuvants during cardiopulmonary bypass. Acta. Anaesthesiol. Scand., v. 44, p. 261-273, 2000.

WADWORTH, A.N.; MURDOCH, D.; BROGDEN, R.N. Atenolol: A reappraisal of its pharmacological proprieties and therapeutic use in cardiovascular disorders. Drugs, v. 42, n. 3, p. 468-510, 1991.

WALLACE, A.; LAYUG, B.; TATEO, I.; LI, J.; HOLLENBERG, M.; BROWNER, W.; MILLER, D.; MANGANO, D.T. Prophylactic atenolol reduces postoperative myocardial ischemia. Anesthesiology, v. 88, n. 1, p. 7-17, 1998.

Recebido para publicação em 03 de outubro de 2005. Aceito para publicação em 09 de março de 2006. 